\title{
Effet de l'interaction entre des modes de gestion de fertilité et la macrofaune sur la productivité du niébé et du sorgho en zone nord soudanienne du Burkina Faso
}

\author{
Jean OUEDRAOGO ${ }^{1 *}$, Elisée OUEDRAOGO ${ }^{2}$ et Hassan Bismarck NACRO ${ }^{1}$ \\ ${ }^{I}$ Institut du Développement Rural, Laboratoire d'Etude, de Recherche sur la Fertilité des Sols (LERF), \\ Université Polytechnique de Bobo-Dioulasso, 01 BP 1091 Bobo-Dioulasso, Burkina Faso. \\ ${ }^{2}$ Banque Mondiale, 01 BP 622 Ouagadougou 01, Burkina Faso. \\ *Auteur correspondant ; E-mail: jeanouedraogo84@yahoo.fr; Tel. (+226) 70634360
}

\section{RESUME}

L'intensification des systèmes de production agricole se traduit par l'utilisation de plus en plus importante des pesticides chimiques de synthèse. La macrofaune du sol est gravement menacée par l'usage de ces pesticides qui réduisent fortement sa diversité et son abondance. Cette étude a été conduite pour évaluer l'impact de l'interaction entre la macrofaune et des modes de gestion de la fertilité des sols sur la productivité du sorgho et du niébé en zone nord soudanienne du Burkina Faso. Le dispositif expérimental comprend deux traitements principaux (parcelles avec et sans macrofaune) et quatre traitements secondaires qui sont de différents modes de gestion de la fertilité du sol. En présence de la macrofaune, le compost a entraîné une augmentation de rendement grain du sorgho de $385 \%$, contre $78 \%$ en absence de la macrofaune. Ce résultat montre clairement l'importance de la macrofaune dans le processus de mise à disposition des nutriments pour les cultures. L'usage exclusif de compost ou la combinaison des matières organiques à $\mathrm{C} / \mathrm{N}$ élevé à l'urée peuvent être vulgarisés dans les systèmes de culture à faible intrants externes. La quantité d'urée à associer au compost pourrait être diminuée et apportée au moment où la plante en a vraiment besoin.

(c) 2014 International Formulae Group. All rights reserved.

Mots clés : rendement, compost, urée, systèmes de production, légumineuses, céréales.

\section{INTRODUCTION}

La croissance de la population en Afrique sub-saharienne est plus rapide que celle de la production agricole. Il en résulte une baisse de la production agricole par personne (FAO, 2001), accentuée par la péjoration climatique. Cette baisse de la production n'est pas seulement une résultante de la croissance démographique et de la péjoration climatique. Elle est aussi une conséquence de la faible fertilité initiale des sols et des mauvaises pratiques agricoles
(FAO, 2001 ; Bationo et al., 2012). Ainsi, la nécessité d'accroître la production agricole pour satisfaire les besoins grandissants de la population s'est traduite par des modifications des systèmes de production dans les agrosystèmes (Masse, 2007). Au Burkina Faso, comme dans la plupart des pays de l'Afrique de l'Ouest, plusieurs modes de gestion de la fertilité incluant l'usage de la matière organique de diverses qualités, des engrais ou l'introduction des légumineuses dans les rotations ont été adoptés (Bado, 
2002 ; Ouédraogo, 2004 ; Bationo et al., 2012). On assiste aussi à une utilisation de plus en plus importante des pesticides chimiques de synthèse, qui est l'un des facteurs de l'intensification agricole. Ces modes de gestion de la fertilité affectent les caractéristiques physico-chimiques et biologiques du sol et, par conséquent, la productivité agricole. De nombreux travaux ont porté sur les effets des différentes pratiques de gestion de la fertilité des sols sur les propriétés chimiques et physiques des sols, et les rendements des cultures (Bonzi, 2002 ; Lompo, 2009 ; Diogo et al., 2010 ; Kiba et al., 2012). La plupart de ces études a surtout porté sur les effets des pratiques de gestion de la fertilité des sols sur les bilans des nutriments (Haileslassie et al., 2006; Tittonell et al., 2007). Il est cependant reconnu qu'une utilisation efficiente des ressources organiques apportées au sol dépend en grande partie de la compréhension du rôle de la faune du sol. Or, très peu d'auteurs se sont intéressés à l'effet des pratiques de gestion de la fertilité des sols sur la pédofaune, qui est pourtant une composante jouant un rôle clé dans les processus de production de nutriments dans le sol. En effet, plusieurs études ont montré que le fonctionnement des écosystèmes tropicaux et sub-tropicaux est influencé par la macrofaune du sol (Six et al., 2002 ; Lavelle et al., 2006). Celle-ci constitue une ressource indispensable à la conservation des sols et à l'amélioration des caractéristiques chimiques et hydrodynamiques (Ouédraogo, 2004 ; Oberson et al., 2006 ; Pey, 2010), et de la production agricole (Blouin et al., 2005). Cependant, la macrofaune est gravement menacée car la plupart des interventions humaines, en particulier l'usage des pesticides de synthèse et le labour, réduisent fortement sa diversité et son abondance (Nonguierma, 2006). Cette étude a été conduite pour évaluer l'effet de l'interaction de la macrofaune et des modes de gestion de la fertilité du sol sur les rendements du sorgho et du niébé. L'évaluation de cette interaction pourrait être une étape importante pour repenser la gestion de la fertilité et les stratégies de protection des cultures, et conduire à la promotion d'une agriculture écologique et durable. L'hypothèse de recherche est que l'usage de la matière organique en présence de la macrofaune augmente la productivité des cultures.

\section{MATERIEL ET METHODES \\ Description du site}

Le dispositif expérimental a été installé sur un sol ferrugineux tropical lessivé profond (CPCS, 1967 ; Lixisols (WRB, 2006)) en zone nord soudanienne du Burkina Faso $\left(12^{\circ} 08^{\prime}\right.$ 02' de latitude Nord et $1^{\circ} 24^{\prime}$ 54' de longitude Ouest). La saison des pluies couvre les mois de juin à septembre avec une pluviosité moyenne de $749 \mathrm{~mm}$ pour les dix dernières années (2003-2012). Les paramètres physico-chimiques de 1'horizon de surface (0$20 \mathrm{~cm}$ ) sont résumés dans le Tableau 1. Il s'agit d'un sol à texture limono-sableuse en surface, acide et pauvre en matière organique (moins de 1\%). Le taux de saturation du complexe absorbant est moyen.

\section{Dispositif expérimental}

Le dispositif expérimental installé au cours de la campagne 2007-2008, un est Splitplot en blocs complètement randomisés à trois répétitions. Il comprend deux (02) traitements principaux (parcelles avec macrofaune et parcelles sans macrofaune), quatre (04) traitements secondaires qui sont les différents modes de gestion de la fertilité du sol (Compost (C) ; Compost + Urée (CU) ; Urée (U); Tiges + Urée (TU)), et une parcelle Témoin (To). La destruction de la macrofaune a été réalisée par application au sol de pesticides chimiques de synthèse: un organophosphoré (Chlorpyrifos-éthyl, connu sous le nom commercial de DADYRSBAN 4 E) appliqué à la dose de 240 g.ha $^{-1}$, et un organochloré (Endosulfan commercialisé sous le nom de CALLIFAN 500 EC) à la dose de 250 g.ha ${ }^{-1}$. L'Endosulfan et le Chlorpyrifoséthyl ont été appliqués à la dose de 1,25 g 
d'Endosulfan et 1,2 g de Chlorpyrifos-éthyl par parcelle élémentaire. Pour ce faire, un mélange contenant $2,5 \mathrm{ml}$ des pesticides CALLIFAN 500 EC et DADYRSBAN 4 E a alors été dilué dans 15 litres d'eau, puis appliqué sur chaque parcelle élémentaire à l'aide d'un pulvérisateur à dos et à pression soutenue. Les applications des pesticides au sol ont été faites au semis, à 35 jours après semis (JAS) et à 80 JAS en 2008-2009. En 2010-2011, les pesticides ont été appliqués au semis, à 23 JAS et à 54 JAS. Deux désherbages ont été réalisés sur chaque culture.

La matière organique (compost et tiges de sorgho) dont les caractéristiques sont présentées dans le Tableau 2 a été appliquée à la dose de 4 tonnes de matières sèches/ha trois jours avant le semis, et l'urée à la dose de 30 $\mathrm{kg} \mathrm{N} / \mathrm{ha}$ au semis. Les parcelles élémentaires ont une superficie de $50 \mathrm{~m}^{2}$ (5 m x $\left.10 \mathrm{~m}\right)$. Une allée principale de $10 \mathrm{~m}$ sépare deux blocs consécutifs. Dans chaque bloc, les traitements principaux sont séparés par des allées secondaires de $5 \mathrm{~m}$, et les traitements secondaires par des allées de $3 \mathrm{~m}$. Toutes les parcelles ont été aménagées en bas de pente par la technique des cordons pierreux.

\section{Conduite de l'expérimentation et évaluation de la production}

Le semis a été précédé d'un labour à la charrue bovine qui a permis d'enfouir les substrats organiques et minéraux dans toutes les parcelles, sauf dans la parcelle témoin où un désherbage très superficiel a été fait. La variété SARIASSO 14 de sorgho avec un rendement potentiel de $5 \mathrm{t} /$ ha a été semée en 2008-2009 et la variété KVX 61-1 de niébé avec un potentiel de $1500 \mathrm{~kg} /$ ha a été semée en 2010-2011. Ce sont des variétés très bien adaptées à la zone nord soudanienne. Le sorgho et le niébé constituent les principales cultures en rotation dans la zone nord soudanienne du Burkina Faso.

Le semis du sorgho et du niébé a été fait suivant des écartements de $80 \mathrm{~cm}$ entre les lignes et $40 \mathrm{~cm}$ sur les lignes. Trois à quatre grains ont été semés par poquet. Un démariage a été réalisé pour ajuster le nombre de plants à deux par poquet. Deux sarclages ont été faits.

L'évaluation de la production du sorgho a été faite sur une parcelle utile de 31,28 $\mathrm{m}^{2}$ délimitée à l'intérieur de chaque parcelle élémentaire. Elle a concerné le rendement grains et la biomasse végétale (pailles, glumes, panicules).

\section{Analyses statistiques}

Les rendements grains et pailles ont été soumis à une analyse de variance (ANOVA), avec le logiciel Genstat version Discovery 4. La séparation des moyennes a été effectuée par le test de Student Newman Keuls au seuil de $5 \%$.

L'interaction entre la macrofaune et les modes de gestion de la fertilité sur le rendement des cultures a été calculée en utilisant la formule utilisée par Ouédraogo et al., 2007 :

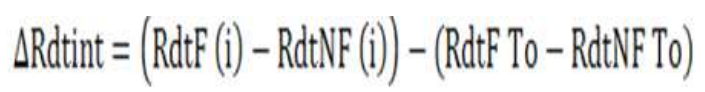

$\Delta$ Rdtint est le l'interaction entre la macrofaune du sol et le mode de gestion de la fertilité ;

RdtF (i) est le rendement dans la parcelle i en présence de la macrofaune du sol;

RdtNF (i) est le rendement dans la parcelle i sans macrofaune du sol;

$\operatorname{Rdt} F$ To, est le rendement de la parcelle témoin avec macrofaune du sol ;

RdtNF To est le rendement de la parcelle témoin sans macrofaune du sol.

\section{RESULTATS}

Effet de la macrofaune et des modes de gestion de la fertilité sur la production du sorgho

Les variations du rendement en fonction du mode de gestion de la fertilité du sol et en présence ou non de la macrofaune du 
sol sont présentées dans le Tableau 3. Les rendements varient de $514 \mathrm{~kg} / \mathrm{ha}$ (Témoin) à 2494 (sol amendé avec du compost) en présence de macrofaune, et de $544 \mathrm{~kg} / \mathrm{ha}$ (Témoin) à $2233 \mathrm{~kg} / \mathrm{ha}$ (sol amendé avec de l'urée) en absence de macrofaune. La plus forte production en grain a été obtenue sur le traitement compost en présence de la macrofaune du sol avec $2494 \mathrm{~kg} / \mathrm{ha}$. Ce traitement diffère significativement du traitement compost en absence de la macrofaune du sol. Les traitements Tiges + Urée (TU) et Compost + Urée (CU) ne diffèrent pas significativement. En présence de la macrofaune du sol, l'usage du compost seul a donné un rendement significativement supérieur à sa combinaison avec l'urée ou à l'urée seul. En effet, une baisse respective de $80 \%$ et $150 \%$ du rendement grain du sorgho a été obtenue en combinant l'urée au compost, ou en l'utilisant exclusivement. Par contre, en absence de la macrofaune du sol, c'est l'inverse qui est observée. Ainsi, la plus forte production a été observée sur le traitement Urée (2233 kg/ha) suivi de la combinaison Compost + Urée (1849 kg/ha). Ces deux traitements sont statistiquement similaires au traitement Compost en présence de la macrofaune du sol. La même tendance a été observée concernant le rendement paille.

En présence de la macrofaune du sol, la combinaison des tiges de sorgho (à $\mathrm{C} / \mathrm{N}$ élevé) à l'urée, a donné de meilleurs rendements grain et paille par rapport à la combinaison Compost + Urée ( $\mathrm{C} / \mathrm{N}$ faible). Cependant, l'effet contraire a été observé en l'absence de la macrofaune du sol.

L'évaluation de l'interaction entre la macrofaune et les modes de gestion de la fertilité sur le rendement des cultures montre alors qu'il y a une interaction positive d'une part, entre la macrofaune et le traitement Compost, et d'autre part, entre la macrofaune et le traitement Tiges + Urée (Figure 1). Par contre, une interaction négative entre l'urée seul ou sa combinaison avec le compost et la macrofaune, a été observée. Le rendement est élevé lorsque le compost est apporté en présence de la macrofaune du sol (1557 $\mathrm{kg} / \mathrm{ha}$ ).

\section{Effet de la macrofaune et des modes de gestion de la fertilité sur la production du niébé}

Les résultats ont montré que les meilleurs rendements graines et fanes ont été obtenus sur les traitements compost (C) et Tiges + Urée (TU) et ce, en présence et en absence de la macrofaune du sol (Tableau 4). Les rendements sont de 835,8 et $827,4 \mathrm{~kg} / \mathrm{ha}$ respectivement pour le sol amendé avec $\mathrm{C}$ et TU en présence de la macrofaune, et de 792,7 et $785 \mathrm{~kg} / \mathrm{ha}$ pour les mêmes traitements en absence de macrofaune. Ces traitements diffèrent significativement des autres traitements. En présence comme en absence de la macrofaune du sol, la combinaison du compost à l'urée a eu un effet dépressif (comparativement aux autres traitements) sur la productivité du niébé. L'application de l'urée seule en présence comme en absence de la macrofaune a donné des rendements comparables à celui du témoin. Le meilleur rendement en fanes a été obtenu avec le traitement compost.

L'analyse de l'interaction entre les modes de gestion de la fertilité des sols et la macrofaune du sol, a révélé une interaction positive mais faible entre la macrofaune et les traitements Compost, Tiges + Urée, et Urée (Figure 2). Seul le traitement Compost + Urée a donné une forte interaction négative. 
Tableau 1 : Caractéristiques physico-chimiques du sol du site d'étude.

\begin{tabular}{lc}
\hline Paramètres mesurés & Valeurs \\
\hline Argile $(\%)$ & 8,67 \\
Limons $(\%)$ & 31,91 \\
Sables $(\%)$ & 59,42 \\
Matière organique totale $(\%)$ & 0,84 \\
Azote total $(\%)$ & 0,04 \\
Phosphore total $(\mathrm{ppm})$ & 85,00 \\
Potassium total $(\mathrm{ppm})$ & 465,67 \\
Potassium disponible $(\mathrm{ppm})$ & 82,27 \\
Somme des bases $(\mathrm{S})(\mathrm{mEq} / 100 \mathrm{~g})$ & 1,52 \\
Capacité d'échange $(\mathrm{T})(\mathrm{mEq} / 100 \mathrm{~g})$ & 2,36 \\
Taux de saturation $(\mathrm{S} / \mathrm{T})(\%)$ & 64,00 \\
pH - eau & 6,06 \\
\hline So &
\end{tabular}

Source : CEAS, 2008

Tableau 2 : Caractéristiques chimiques des tiges et du compost.

\begin{tabular}{lcccc}
\hline \multirow{2}{*}{ Paramètres mesurés } & Compost & Tiges & Compost & Tiges \\
\cline { 2 - 5 } & \multicolumn{2}{c}{$\mathbf{2 0 0 8}$} & & $\mathbf{2 0 1 0}$ \\
\hline $\begin{array}{l}\text { Matière organique } \\
\text { totale \% }\end{array}$ & $21,7 \pm 1,23$ & $94,19 \pm 0,3$ & $19,80 \pm 5,02$ & $96,25 \pm 0,35$ \\
\hline $\begin{array}{l}\text { Azote total \% } \\
\text { C/N }\end{array}$ & $1,5 \pm 0,5$ & $1,27 \pm 0,2$ & $0,54 \pm 0,03$ & $0,20 \pm 0,05$ \\
\hline $\begin{array}{l}\text { Phosphore total \% } \\
\left(\mathrm{P}_{2} \mathrm{O}_{5}\right)\end{array}$ & $0,4 \pm 0,1$ & 44 & 21 & 278 \\
\hline $\begin{array}{l}\text { Potassium total \% } \\
\left(\mathrm{K}_{2} \mathrm{O}\right)\end{array}$ & $0,8 \pm 0,3$ & $2,2 \pm 0,5$ & $0,32 \pm 0,02$ & $0,68 \pm 0,15$ \\
\hline $\mathrm{pH}$ eau & $7,5 \pm 0,0$ & - & $7,26 \pm 0,18$ & \\
\hline \multicolumn{1}{c}{ Source : CEAS, 2008 et 2010} & & $0,39 \pm 0,03$ & \\
\hline
\end{tabular}


Tableau 3 : Effet de la macrofaune et des modes de gestion de la fertilité sur le rendement grain et paille du sorgho.

\begin{tabular}{|c|c|c|c|c|}
\hline \multirow[b]{2}{*}{ Traitements } & \multicolumn{2}{|c|}{ Rendement grain (kg/ha) } & \multicolumn{2}{|c|}{ Rendement paille (kg/ha) } \\
\hline & $\begin{array}{c}\text { Avec } \\
\text { macrofaune }\end{array}$ & Sans macrofaune & Avec macrofaune & Sans macrofaune \\
\hline Compost & $2494^{\mathrm{a}}$ & $967^{\mathrm{cd}}$ & $9540^{\mathrm{a}}$ & $7157^{\mathrm{bcd}}$ \\
\hline Compost + Urée & $1383^{\mathrm{bcd}}$ & $1849^{\mathrm{abc}}$ & $6353^{\mathrm{d}}$ & $8672^{\mathrm{abc}}$ \\
\hline Tiges + Urée & $2401^{\mathrm{ab}}$ & $1464^{\mathrm{abcd}}$ & $8899^{\mathrm{ab}}$ & $7828^{\text {abcd }}$ \\
\hline Urée & $997^{\mathrm{cd}}$ & $2233^{\mathrm{ab}}$ & $6693^{\mathrm{cd}}$ & $7751^{\text {abcd }}$ \\
\hline Témoin & $514^{\mathrm{d}}$ & $544^{\mathrm{d}}$ & $5727^{\mathrm{d}}$ & $6445^{\mathrm{d}}$ \\
\hline Probabilité & \multicolumn{2}{|r|}{0,016} & \multicolumn{2}{|c|}{0,044} \\
\hline Signification & \multicolumn{2}{|r|}{ S } & \multicolumn{2}{|c|}{$\mathrm{S}$} \\
\hline
\end{tabular}

Tableau 4 : Effet de la macrofaune et des modes de gestion de la fertilité sur le rendement grain et fanes du niébé.

\begin{tabular}{lcccc}
\hline \multirow{2}{*}{ Traitements } & \multicolumn{2}{c}{ Rendement grain (kg/ha) } & \multicolumn{2}{c}{ Rendement fanes (kg/ha) } \\
\cline { 2 - 5 } & $\begin{array}{c}\text { Avec } \\
\text { macrofaune }\end{array}$ & $\begin{array}{c}\text { Sans } \\
\text { macrofaune }\end{array}$ & $\begin{array}{c}\text { Sans } \\
\text { Avec macrofaune }\end{array}$ \\
\hline macrofaune \\
\hline Compost & $835,8^{\mathrm{a}}$ & $792,7^{\mathrm{a}}$ & $1842^{\mathrm{a}}$ & $1719^{\mathrm{ab}}$ \\
Tiges + Urée & $448,1^{\mathrm{c}}$ & $577,6^{\mathrm{b}}$ & $914^{\mathrm{c}}$ & $1431^{\mathrm{b}}$ \\
Urée & $827,4^{\mathrm{a}}$ & $785^{\mathrm{a}}$ & $1727^{\mathrm{ab}}$ & $1459^{\mathrm{ab}}$ \\
Témoin & $435,6^{\mathrm{c}}$ & $382,8^{\mathrm{cd}}$ & $969^{\mathrm{c}}$ & $742^{\mathrm{c}}$ \\
\hline Probabilité & $397,7^{\mathrm{cd}}$ & $361,3^{\mathrm{d}}$ & $868^{\mathrm{c}}$ & $969^{\mathrm{c}}$ \\
Signification & \multicolumn{3}{c}{0,047} \\
\hline \multicolumn{1}{c}{ Dans chaque groupe (rendement grain et rendement paille), les moyennes suivies de la même lettre ne diffère pas au } \\
\multicolumn{2}{c}{ seuil de 5\% ; S : significatif ; HS : hautement significatif. }
\end{tabular}




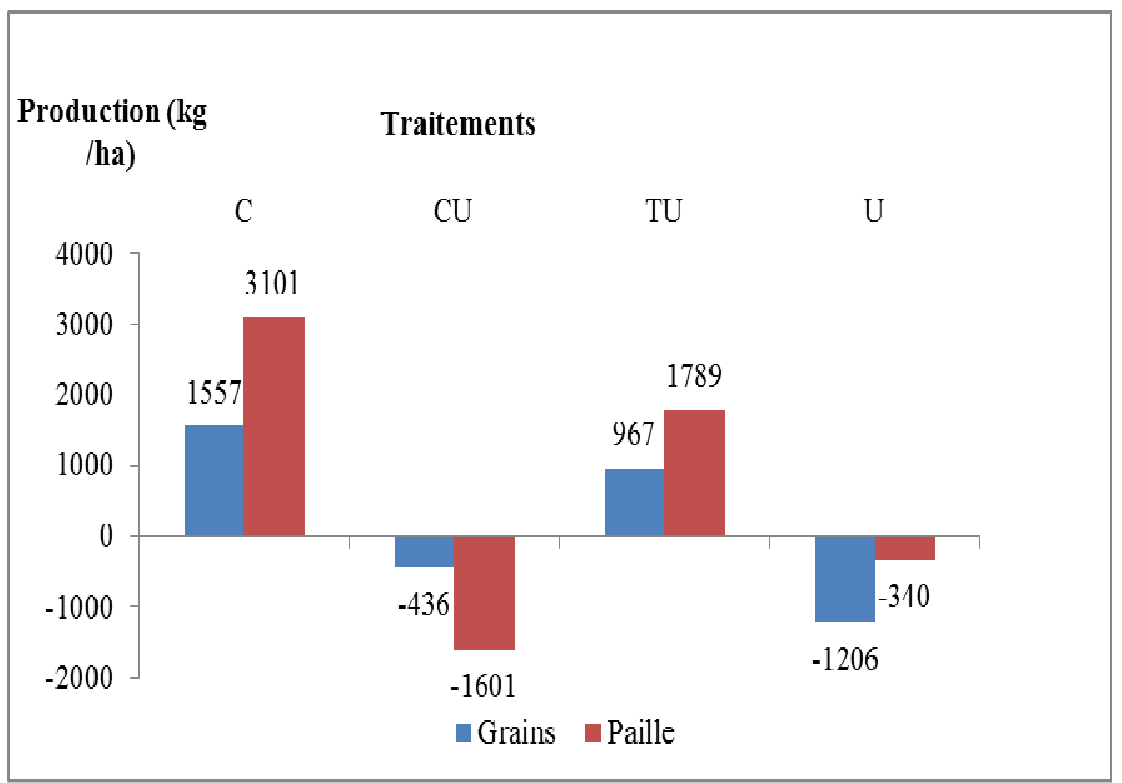

Figure 1 : Interaction entre les modes de gestion de la fertilité et la macrofaune sur les rendements grain et paille du sorgho. C : Compost ; CU : Compost + Urée ; TU : Tiges + Urée ; U : Urée ; To : Témoin.

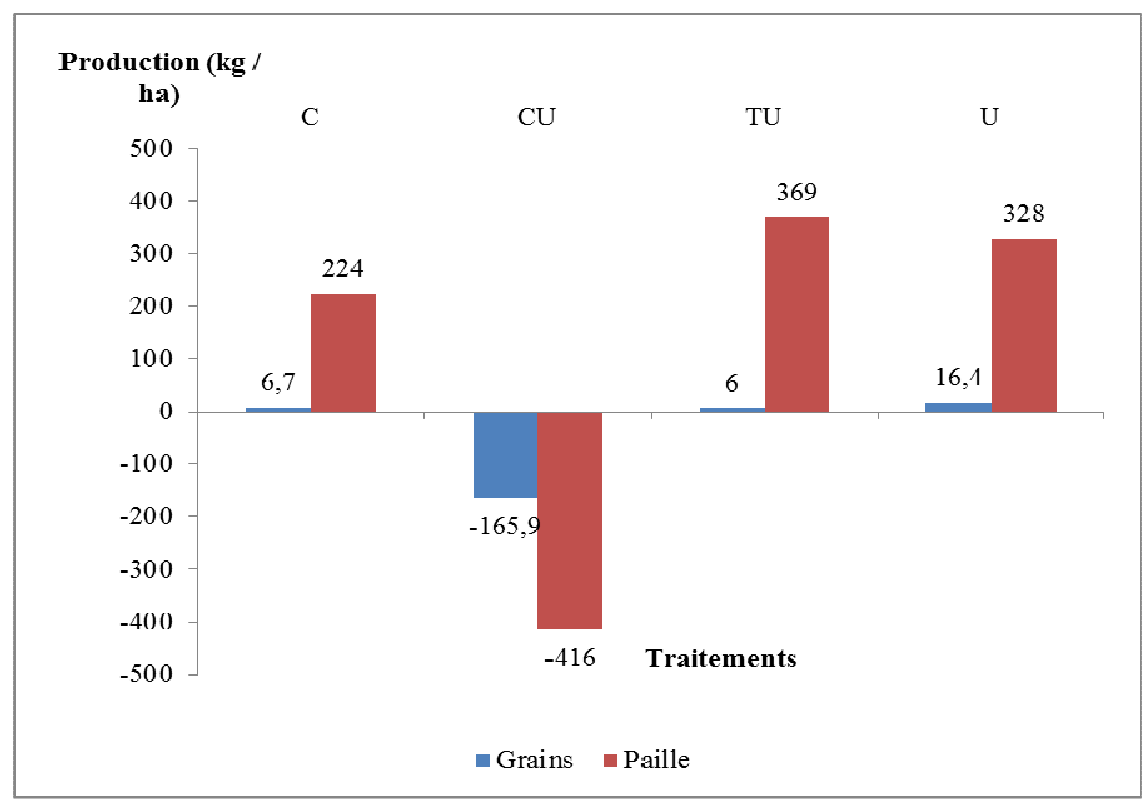

Figure 2 : Interaction entre les modes de gestion de la fertilité et la macrofaune sur les rendements grain et paille du niébé. C : Compost ; CU : Compost + Urée ; TU : Tiges + Urée ; U : Urée ; To : Témoin. 


\section{DISCUSSION}

Interaction entre les modes de gestion de la fertilité et la macrofaune sur la productivité du sorgho et du niébé

Les résultats ont révélé un faible niveau de productivité du sorgho et du niébé dans le témoin et le traitement avec usage exclusif de l'urée. Ces résultats s'expliquent par le faible niveau de fertilité initiale des sols. En effet, plusieurs études réalisées en Afrique de l'Ouest ont incriminé ce facteur comme l'une des causes majeures de la faible productivité agricole (Cattan et al., 2001 ; Ouattara et al., 2006; Koulibaly et al., 2010). L'usage exclusif d'urée, ou la pratique d'agriculture extensive sans apport de fertilisant, conduit à une dégradation des caractéristiques chimiques, physiques et biologiques des sols. C'est pourquoi une interaction négative entre la macrofaune et l'usage exclusif d'urée a été observé sur la production du sorgho. En augmentant la vitesse de minéralisation de la matière organique (Ouédraogo et al., 2007), l'urée réduit d'une part la quantité d'énergie disponible pour la macrofaune et d'autre part, entraîne des pertes de nutriments, impactant négativement la production du sorgho.

Une interaction positive sur la productivité du sorgho et du niébé a été obtenue entre la macrofaune et le compost, et entre la macrofaune et la combinaison des tiges de sorgho à l'urée. L'apport de la matière organique, support de la vie du sol permet d'augmenter la productivité du sorgho et du niébé en présence de la macrofaune. Ces résultats sont en accord avec les observations de Lavelle et al. (2006), qui indiquent que la présence de la macrofaune entraîne une augmentation de la production agricole grâce à son rôle dans l'amélioration des propriétés chimiques, physiques et biologiques du sol. Par contre, une interaction négative a été obtenue entre la macrofaune et la combinaison du compost à l'urée sur la productivité du sorgho et du niébé. L'usage combiné de la matière organique de bonne qualité à l'urée, a entrainé une baisse de la productivité du sorgho et du niébé. Ces résultats corroborent ceux obtenus par Mando et al. (2005). L'ajout d'urée en présence de la macrofaune, accélère la vitesse de minéralisation du compost (Ménard, 2005 ; Ouédraogo et al., 2007). La baisse constatée des rendements du sorgho et du niébé consécutive à l'usage combiné de la matière organique et de l'urée, pourrait s'expliquer par la mauvaise synchronisation entre la minéralisation de la matière organique et les besoins des cultures.

Par contre, un effet positif de la combinaison de la matière organique à l'urée a été observé sur le traitement TU, corroborant les résultats obtenus par Bababe (1998), Mando et al. (2005) et Niang (2006). Les travaux de Ouédraogo et al. (2006) ont également montré que la combinaison de matière organique de pauvre qualité $(\mathrm{C} / \mathrm{N}$ élevé) à l'urée permet d'accroître l'efficience de l'utilisation de l'eau et des nutriments par le sorgho. L'enfouissement des pailles stimule le développement racinaire (Bababe, 1998), accroissant ainsi le volume de sol exploré par les racines. Les rendements sont toujours plus élevés en cas de fertilisation organique (compost) ou organo-minérale (Tige+Urée) en présence de la macrofaune du sol. Ceci montre clairement que la présence de la macrofaune est nécessaire pour la décomposition rapide de la matière organique (Ouédraogo et al., 2004) et donc, pour la mise à disposition des nutriments pour les cultures. Selon Marhan (2004), la digestion de la litière par les vers de terre entraîne une augmentation de la biomasse et du nombre des champignons. C'est ce qui explique sans doute l'accroissement du rendement grain en présence de la macrofaune même lorsque la source organique (tige) est connue pour son faible potentiel de minéralisation.

\section{Conclusion}

L'usage du compost et la combinaison des tiges à l'urée ont été les deux meilleurs 
modes de gestion de la fertilité des sols. Un effet positif de l'interaction de ces modes de gestion de la fertilité des sols avec la macrofaune a été observé. En effet, avec les traitements Compost, et Tiges + Urée, une augmentation de rendement grain du sorgho de $385 \%$ et $367 \%$, et du niébé de $110 \%$ et $108 \%$ a été observée par rapport au témoin en présence la macrofaune du sol. Par ailleurs, la combinaison de l'urée au compost interagit négativement avec la macrofaune sur le rendement grain du niébé et du sorgho. Dans les systèmes de culture à faibles intrants externes, la réduction de l'usage des pesticides par la promotion de pratiques agroécologiques permettra de préserver la macrofaune du sol et de ce fait, d'améliorer la productivité des cultures.

\section{REMERCIEMENTS}

Cette étude a été réalisée grâce à l'appui financier de la Fondation Internationale de Science (IFS) et à la contribution du Centre Ecologique Albert Schweitzer du Burkina.

\section{RÉFÉRENCES}

Bababe B. 1998. Crop residue application systems and efficiency of water use by pearl millet in northern Nigeria. In Soil Fertility Management in West African Land Use Systems, Renard G, Neef A, Becker K, Von Oppen M. (eds). Proceeding of the regional workshop university of Hohenheim, ICRISAT sahelian centre and INRAN, 4-8 march 1997, Niamey, Niger. Margraf Verlag, Germany, 61-66.

Bado BV. 2002. Rôle des légumineuses dans la fertilité des sols ferrugineux tropicaux des zones guinéennes et soudaniennes du Burkina Faso. Thèse de doctorat, Département des Sols et Environnement, Université Laval, p. 145.

Bationo A, Waswa B, Abdou A, Bado BV, Bonzi M, Iwuafor E, Kibunja C, Kihara J,
Mucheru M, Mugendi D, Mugwe J, MwaleC, Okeyo J, Olle A, Roing K, Sedogo M. 2012. Overview of long term experiments in Africa. In Lessons Learned from Long Term Soil Fertility Management Experiments in Africa, Bationo A, Waswa B, Kihara J, Adolwa I, Vanlauwe B, Saidou K (eds). Springer; 126.

Blouin M, Zuily-Fodil Y, Pham-Thi A, Laffray D, Reversat G, Pando A, Tondoh J, Lavelle P. 2005. Belowground organism activities affect plant aboveground phenotype, inducing plant tolerance to parasites. Ecol. Lett., 8: 202208.

Bonzi M. 2002. Evaluation et déterminisme du bilan de l'azote en sols cultivés du centre Burkina Faso: Etude par traçage isotopique N15 au cours d'essais en station et en milieu paysan. Thèse de Doctorat Unique en Sciences Agronomique, INPL/ENSAIA, Nancy, France, p. 177.

Cattan P, Letourmy P, Zagré B, Minougou A, Compaoré E. 2001. Rendement de l'arachide et du sorgho en rotation sous différents itinéraires techniques au Burkina Faso. Cah. Agric., 10(3): 159172.

CPCS (Commission de pédologie et de cartographie des sols). 1967. Classification des Sols. ENSA: Grignon; 77.

Diogo RVC, Buerkert A, Schlecht E. 2010. Horizontal fluxes and food safety in urban and peri-urban vegetable and millet cultivation of Niamey, Niger. Nutr. Cycl. Agroecosyst., 87: 81-102.

FAO. 2001. Soil Fertility Management in Support of Food Security in Sub-Saharan Africa. FAO: Rome, Italy; 55.

Haileslassie A, Priess JA, Veldkamp E, Lesschen JP. 2006. Smallholders' soil fertility management in the Central Highlands of Ethiopia: implications for 
nutrient stocks, balances and sustainability of agroecosystems. Nutr. Cycl. Agroecosyst., 75: 135-146.

Kiba DI, Lompo F, Compaoré E, Randriamanantsoa L, Sedogo PM, Frossard E. 2012. A decade of non-sorted solid urban waste inputs safely increases sorghum yield in the periurban areas of Burkina Faso. Acta Agriculturae Scandinavica Section B-Soil Plant Sci., 62(1): 59-69.

Koulibaly B, Traoré O, Dakuo D, Zombré PN, Bondé D. 2010. Effet de la gestion des résidus de récolte sur les rendements et les bilans culturaux d'une rotation cottonmaïs-sorgho au Burkina Faso. Tropicultura, 28(3): 184-189.

Lavelle P, Decaëns T, Aubert M, Barot S, Blouin M, Bureau F, Margerie P, Mora P, Rossi JP. 2006. Soil invertebrates and ecosystem services. Eur. J. Soil Biol., 42: S3-S15.

Lompo F. 2009. Effets induits des modes de gestion de la fertilité sur les états du phosphore et la solubilisation des phosphates naturels dans deux sols acides du Burkina Faso, thèse doctorat d'Etat, Université de Cocody, Côte d'Ivoire, p. 254.

Mando A, Bonzi M, Wopereis MCS, Lompo F, Stroosnijder L. 2005. Long-term effects of mineral and organic fertilization on soil organic matter fractions and sorghum yield under Sudano-Sahelian conditions. Soil Use and Manage., 21: 396-401.

Marhan S. 2004. Effects of earthworms on stabilisation and mobilisation of soil organic matter. Dissertation, Fachbereich Biologie der Technischen Universität Darmstadt, p. 160.

Masse D. 2007. Changements d'usage des terres dans les agro-systèmes d'Afrique subsaharienne. Propriétés des sols et dynamique des matières organiques. Mémoire pour l'obtention du diplôme
d'Habilitation à Diriger des Recherches, Ecole Nationale Supérieure Agronomique de Toulouse, France, p. 82.

Ménard O. 2005. Les ouvriers du sol et les pratiques agricoles de conservation. Colloque en Agroenvironnement: «des outils d'intervention à notre échelle ». Centre de référence en agriculture et agroalimentaire du Québec, Québec, p. 6.

Niang D. 2006. Fonctionnement hydrique de différents types de placages sableux dans le sahel Burkinabè. Thèse de Doctorat ès Sciences. Faculté de l'environnement naturel, architectural et construit. École polytechnique fédérale de Lausanne, Suisse, p. 136.

Nonguierma GB. 2006. Contribution à l'évaluation des effets de l'utilisation des pesticides en production maraîchère dans la plaine périurbaine de BoulmiougouOuagadougou. Mémoire de Licence Professionnelle en Génie de l'Environnement. Université de Ouagadougou, Unité de Formation et de Recherche en Sciences Exactes et Appliquées. Institut de Génie de l'Environnement et du Développement durable, Burkina Faso, p. 45.

Oberson A, Bünemann EK, Friesen DK, Rao IM, Smithson PC, Turner BL, Frossard E. 2006. Improving Phosphorus Fertility in Tropical Soils through Biological Interventions. In Improving Phosphorus Fertility in Tropical Soils, Uphoff N, Ball AS, Fernandes E, Herren H, Husson O, Lang M, Palm C, Pretty J, Sanchez P, Sanginga N, Thies J (eds). CRP Press; 532-546.

Ouattara B, Ouattara K, Serpentié G, Mando A, Sédogo MP, Bationo A. 2006. Intensity cultivation induced effects on soil organic carbon dynamic in the western cotton area of Burkina Faso. Nutr. Cycl. Agroecosyst., 76: 331-339.

Ouédraogo E, Brussaard L, Stroosnijder L. 2007. Soil macrofauna and organic 
amendment interactions affects soil carbon and crop performance in semi-arid West Africa. Biol. Fert. Soils, 44(2): 343351.

Ouédraogo E, Mando A, Brussaard L. 2004. Soil macrofaunal-mediated organic resource disappearance in semi-arid West Africa. Appl. Soil Ecol., 27: 259-267.

Ouédraogo E. 2004. Soil quality improvement for crop in semi-arid West Africa. PHD Thesis. University and research centre, Wageningen, The Netherland, p. 193

Pey B. 2010. Contribution de la faune du sol au fonctionnement et à l'évolution des technosols. Thèse en Sciences Agronomiques, Université Nationale Polytechnique de Lorraine, France, p. 254.
Six J, Feller C, Denef K, Gle Sm, Sa Jcm, Albrecht A. 2002. Soil organic matter, biota and aggregation in temperate and tropical soils - Effects of no-tillage. Agronomie, 22: 755-775.

Tittonell P, Vanlauwe B, Ridder N, Giller KE. 2007. Heterogeneity of crop productivity and resource use efficiency within smallholder Kenyan farms: Soil fertility gradients or management intensity gradients? Agr. Syst., 94: 376-390.

WRB (World Reference base for Soil Resources). 2006. A Framework for International Classification, Correlation and Communication. FAO : Rome, Italie ; 116. 